\title{
CONCORDÂNCIA DOS RESULTADOS DO SISTEMA BD PHOENIX COM PROVAS BIOQUÍMICAS MANUAIS NA IDENTIFICAÇÃO DE ENTEROBACTÉRIAS EM AMOSTRAS CLÍNICAS*
}

\author{
AgreEMENT of RESULTS of the BD PhoENIX SySteM \\ AND MANUAL BIOCHEMICAL TESTS IN THE IDENTIFICATION OF \\ ENTEROBACTERIACEAE FROM CLINICAL SAMPLES
}

Natacha Allgayer ${ }^{1}$, Helena Schirmer², Jussara Amaro Castelan ${ }^{3}$

\section{RESUMO}

Introdução: Enterobactérias pertencem a um grupo grande e heterogêneo de bacilos Gram-negativos cujo habitat natural é o cólon de humanos e animais, sendo microrganismos amplamente distribuídos na natureza. Devido a essas características são frequentemente responsáveis por infecções hospitalares. O principal objetivo desse estudo foi avaliar a concordância entre as provas bioquímicas manuais e o sistema automatizado BD Phoenix 100 na identificação de enterobactérias, a partir da análise de registros de pacientes hospitalizados.

Métodos: No período de agosto de 2011 a abril de 2012, foram realizados 303 exames no Laboratório Exame de Análises Clinicas pelos métodos manual e automatizado, os quais foram submetidos a análise dos dados.

Resultados: Do total, $27,7 \%$ foram positivos para enterobactérias. Os microrganismos mais frequentes isolados foram a Klebsiella pneumoniae $(36,9 \%)$ e Escherichia coli $(29,8 \%)$. O estudo apresentou uma alta concordância entre os métodos, principalmente na identificação dos gêneros, caso em que a concordância chegou a $97 \%$ dos registros. Na identificação das espécies, as bactérias dos gêneros Klebsiella spp. e Serratia spp. não tiveram suas espécies identificadas pelo método manual em sete dos registros observados, sendo identificadas somente pela automação. Da mesma forma, algumas amostras de Escherichia coli não foram detectadas pelo método manual, que as identificou como indeterminadas em quatro dos registros analisados.

Conclusão: Verificou-se uma boa concordância entre os métodos na identificação das principais enterobactérias isoladas de amostras clínicas.

Palavras-chave: Enterobactérias; automação; infecções hospitalares

\section{ABSTRACT}

Introduction: Enterobacteriaceae are microorganisms that are widely distributed in nature and belong to a large and heterogeneous group of Gram-negative bacilli whose natural habitat is the colon of human beings and animals and are. Because of these characteristics they are often responsible for hospital infections. The main objective of this study was to assess the agreement between manual biochemical tests and the BD Phoenix 100 automated system in the identification of the Enterobacteriaceae, based on an analysis of hospitalized patients' records.

Methods: In the period between August 2011 and April 2012, 303 tests were made at Laboratório Exame de Análises Clinicas by the manual and automated methods, which were subjected to analysis.

* Laboratório Exame de Análises Clínicas Ltda., Novo Hamburgo - Rio Grande do Sul, Brasil.

Clin Biomed Res. 2015;35(1):43-48

1 Programa de Pós-graduação em Biologia Celular e Molecular Aplicada à Saúde, Universidade Luterana do Brasil (ULBRA). Canoas, RS, Brasil.

2 Universidade Feevale. Novo Hamburgo, RS, Brasil.

3 Laboratório Exame de Análises Clínicas Ltda. Novo Hamburgo, RS, Brasil.

Autor correspondente:

Natacha Allgayer

E-mail: natachaallgayer@gmail.com Universidade Luterana do Brasil Av. Farroupilha, $n^{\circ} 8001$, Sede Campus Canoas.

92425-900, Canoas, RS, Brasil. 
Results: Results revealed that $27.7 \%$ of the tests were positive for Enterobacteriaceae. The most commonly isolated microorganisms were Klebsiella pneumoniae (36.9\%) and Escherichia coli (29.8\%). The study showed a high agreement between the methods, especially in the identification of genders, in which case agreement reached $97 \%$ of records. When it comes to identifying the species, seven samples of Klebsiella spp. and Serratia spp. bacteria had their species identified only by the automated method and not by the manual method. Similarly, some samples of Escherichia coli, were not detected by the manual, which identified them as indeterminate in four of the records analyzed.

Conclusions: There was a good agreement between both methods in identifying the major Enterobacteriaceae isolated from clinical samples.

Keywords: Enterobacteriaceae; automation; hospital infections

Os bacilos Gram-negativos que pertencem à família Enterobacteriaceae são os mais frequentemente isolados a partir de amostras clínicas, sendo responsáveis por qualquer tipo de doença infecciosa. Correspondem a quase $50 \%$ dos casos de sepse e a mais de $70 \%$ das infecções do trato urinário, além de responder por um percentual significativo das infecções intestinais. Atingem principalmente pacientes imunocomprometidos ou debilitados, altamente suscetíveis a infecções hospitalares, quer por colonização por cepas ambientais ou por procedimentos invasivos, como o cateterismo, broncoscopia, ou biopsias cirúrgicas ${ }^{1}$.

Enterobactérias, com poucas exceções, demonstram as seguintes características fisiológicas e bioquímicas: crescem em meios simples, fermentam glicose, são oxidase negativas e reduzem nitratos a nitritos ${ }^{1,2}$.

A maioria dos laboratórios utiliza, como rotina para identificação desses microrganismos, o método manual, baseado em compostos bioquímicos como glicose, lactose, sacarose, citrato, ornitina, lisina, sulfato de hidrogênio, ureia, fenilalanina, entre outros, incorporados a meios contendo ágar ${ }^{3}$. Porém, nos últimos anos os laboratórios de análises clínicas, principalmente os inseridos em ambientes hospitalares, estão optando por usufruir de automações, devido à crescente pressão de médicos e administradores para fornecer resultados de identificação bacteriana rápidos e precisos ${ }^{4}$.

Atualmente um dos sistemas automatizados para a identificação bacteriana disponíveis no mercado é o BD Phoenix 100, que é constituído por painéis descartáveis que combinam identificação e perfil de sensibilidade, com um instrumento que executa a leitura automática, tendo um tempo máximo de 12 horas para obter uma identificação e de 16 horas para completar um antibiograma ${ }^{5}$. Para a identificação de bactérias Gram-negativas, o painel possui 45 substratos bioquímicos $\operatorname{secos}^{6}$, permitindo a discriminação de aproximadamente 160 grupos taxonômicos diferentes ${ }^{5}$.
Sabe-se que tanto os testes bioquímicos manuais quanto os automatizados possuem deficiências na identificação. Essa deficiência pode ser observada na identificação dos gêneros Shigella spp. e Salmonella spp., que requerem testes adicionais por aglutinação ${ }^{4}$, e na diferenciação entre os gêneros Klebsiella spp. e Enterobacter spp. Nem todas as espécies de Enterobacter spp. são móveis, e como tal podem ser erroneamente identificadas como Klebsiella spp., tornando-se necessário o uso de um meio com ornitina descarboxilase para a diferenciação ${ }^{7}$. Sendo assim, o objetivo deste trabalho foi verificar a concordância entre os resultados obtidos por ambos os métodos, manual e automatizado, a partir de amostras clínicas provenientes de um ambiente hospitalar, encaminhadas a um laboratório de análises clínicas.

\section{MÉTODOS}

Foram analisados 303 registros oriundos de pacientes hospitalizados que tiveram suas amostras submetidas a análise bacteriológica, atendidos no Laboratório Exame de Análises Clínicas Ltda., em Novo Hamburgo (RS), Brasil, no período de agosto de 2011 a abril de 2012. Para o estudo de concordância foram selecionados registros de pacientes que tiveram suas amostras identificadas com a presença de enterobactérias por testes bioquímicos manuais e pelo sistema automatizado BD Phoenix 100.

Em todas as 303 amostras foi realizada a técnica de Gram. Amostras com presença de bacilos Gram-negativos passaram para a etapa seguinte de identificação. Tanto para o método manual como para o automatizado foi realizado o cultivo primário de 24 horas em ágar sangue ou ágar cromogênico. $O$ ágar cromogênico foi utilizado como meio de cultivo apenas em amostras de urina. Após o crescimento de culturas puras a identificação por ambos os métodos foi realizada. No método manual, foi utilizado um conjunto de meios bioquímicos, contemplando seis tubos: TSI (Tríplice Açúcar Ferro), citrato, MIO (Motilidade, Indol, Ornitina), SIM (Sulfeto, Indol, 
Motilidade), fenilalanina e ureia. Para a identificação no método automatizado foram realizadas escalas de 0,5 McFarland, em meios líquidos de ID Phoenix padronizados. Essas escalas foram colocadas em painéis NMIC/ID 121 e carregadas no equipamento.

Os resultados foram expressos em percentual, para análise da prevalência de enterobactérias e das amostras clínicas com a presença destas, e submetidos ao teste kappa para avaliar a intensidade de concordância entre os métodos manual e automatizado.

\section{RESULTADOS}

Entre os 303 registros avaliados, $84(27,7 \%)$ foram positivos para enterobactérias. Na categoria de não enterobactérias, correspondente a 188 (62\%) dos registros, foram identificados $105(55,9 \%)$ cocos Gram-positivos, 67 (35,6\%) bacilos Gram-negativos não fermentadores, $16(8,5 \%)$ foram outros patógenos (Corynebacterium spp. e Aeromonas spp.). Além disso, foram encontrados $31(10,2 \%)$ resultados negativos, nos quais não foram identificados microrganismos.

Nos 84 registros com presença de enterobactérias, foi realizada uma análise de concordância entre os métodos manual (bioquimismo) e o automatizado (BD Phoenix). O coeficiente de concordância kappa para gênero foi 0,971 (IC95\% 0,943-0,998), enquanto que para gênero e espécie esse coeficiente foi 0,834 (IC95\% 0,746-0,922). Ambos resultados foram significativos $(P<0,001)$. Do total de amostras analisadas, em apenas 11 registros não foi observada concordância em nível de gênero e espécie entre os métodos. Destas, quatro foram identificadas apenas ao nível de gênero pelo método manual e como três Klebsiella pneumoniae e uma Klebsiella oxytoca pelo método automatizado. Três amostras foram identificadas como Serratia spp. pelo método manual e como Serratia marcescens pelo método automatizado, e quatro amostras foram consideradas como indeterminadas pelo método manual e identificadas como Escherichia coli pelo método automatizado (tabela 1).

As outras enterobactérias foram identificadas ao nível de gênero e espécie por ambos os métodos. Na Figura 1 observa-se uma predominância das bactérias Klebsiella pneumoniae e Escherichia coli, com $31(36,9 \%)$ e $25(29,8 \%)$ identificações, respectivamente, nas amostras de pacientes hospitalizados. De acordo com a Figura 2, o material que apresentou maior prevalência na identificação de enterobactérias foi a urina (25\%), seguido por amostras de sangue $(23,8 \%)$.

\section{DISCUSSÃO}

Alguns estudos vêm demonstrando a eficácia e a reprodutibilidade dos sistemas automatizados na rotina dos laboratórios de microbiologia. Em nosso estudo procuramos estabelecer a concordância entre

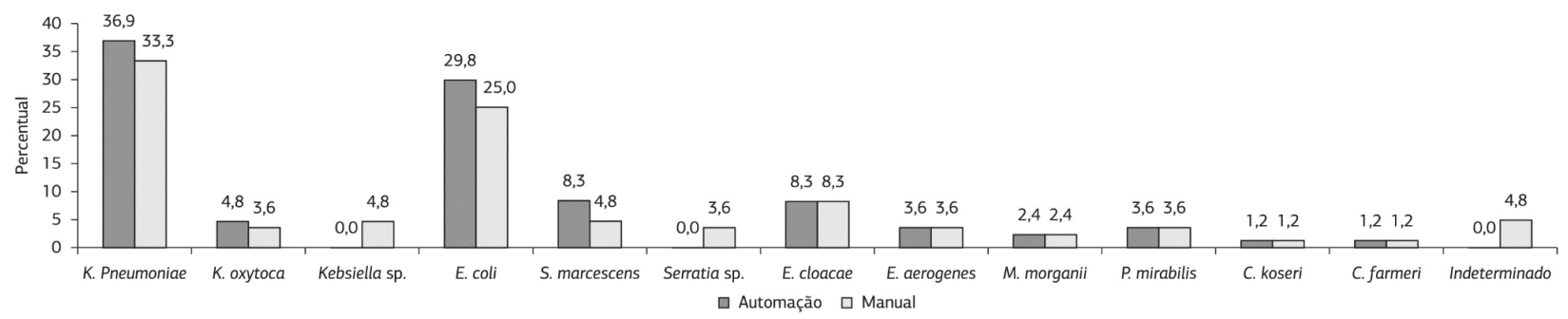

Figura 1: Detecção de enterobactérias em 303 amostras clínicas avaliadas de acordo com o método manual (bioquimismo) e com o método automatizado (BD Phoenix).

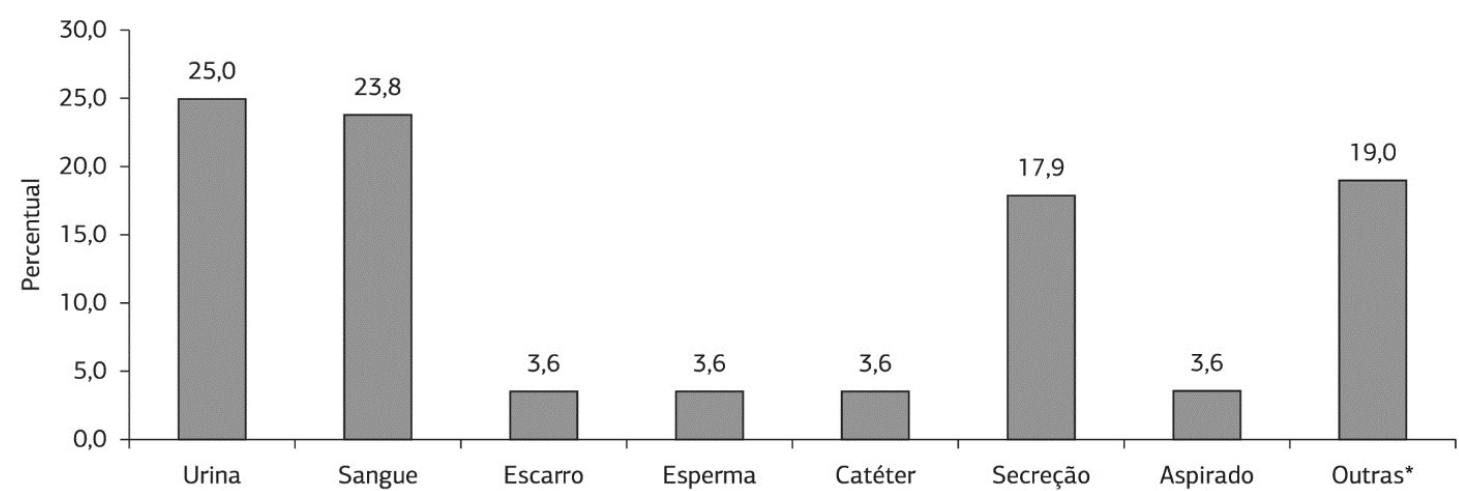

Figura 2: Frequência de amostras clínicas com a presença de enterobactérias*: abcessos, úlceras, biópsias, fezes e lesões diversas. 


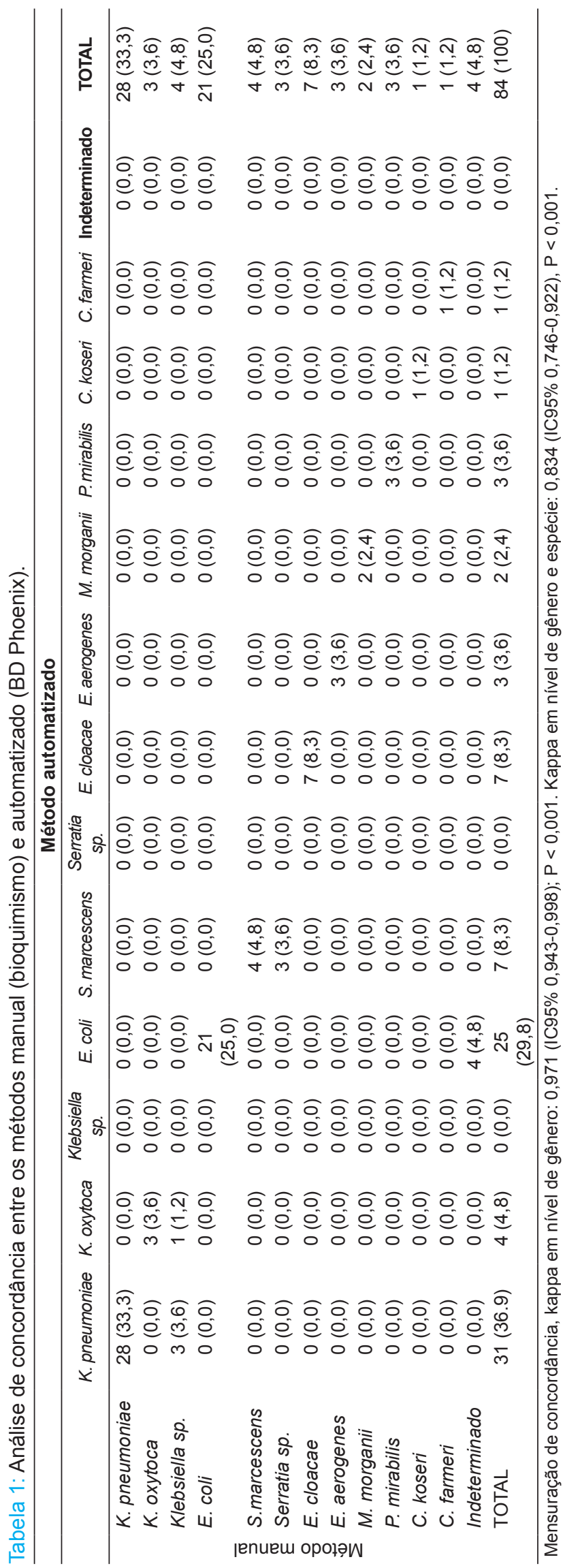


esse método e o método manual. Ambos os métodos mostraram-se eficazes na identificação da maioria das enterobactérias, sendo encontrado um nível de concordância maior em relação à identificação dos microrganismos ao nível de gênero do que ao nível de espécie.

Resultado similar ao acima citado foi encontrado no estudo de Vargas et al. $^{8}$, que obteve um nível de concordância de $98,1 \%$ entre os métodos para a identificação de espécies e de $99,5 \%$ ao nível de gênero, em amostras clínicas de pacientes hospitalizados.

Segundo Snyder et al. ${ }^{4}$, a precisão do equipamento BD Phoenix na identificação de enterobactérias em gênero e espécie, foi determinada como sendo de $100 \%$, em comparação com testes bioquímicos manuais. Já no estudo de O'Hara ${ }^{9}$, apenas $89,9 \%$ das culturas da família Enterobacteriaceae foram identificadas corretamente nos níveis de gênero e espécie pela automação, quando comparada ao método manual. Os erros da automação observados neste estudo foram relacionados, em sua maioria, ao gênero Salmonella spp. ${ }^{4,9}$

Stefaniuk et al. ${ }^{10}$, comparando o sistema Phoenix com um método de identificação manual, obtiveram uma precisão de $92,5 \%$ para a família Enterobacteriaceae. As discordâncias ocorreram ao nível de gênero em três isolados de enterobactérias, identificados pelo método manual como dois isolados de Proteus mirabilis e um isolado de Serratia marcescens, que no Phoenix foram identificados como Pasteurella multocida, Alcaligenes faecalis, e Pantoea agglomerans, respectivamente ${ }^{10}$.

O estudo de Carroll et al. ${ }^{3}$, com 251 isolados de enterobactérias identificados por ambos os métodos, manual e BD Phoenix, obteve uma concordância de 95,6\% em gênero e 94,4\% em nível de espécie. Erros preocupantes observados nos resultados obtidos pelo método automatizado foram uma Escherichia coli identificada como Salmonella spp. e quatro amostras com Shigella spp. identificadas como Escherichia coli, além de duas cepas de Klebsiella pneumoniae identificadas com Klebsiella oxytoca e três erros ao nível de espécie do gênero Enterobacter spp. ${ }^{3}$. Esse estudo apresentou uma concordância similar à do presente estudo.

De acordo com os resultados anteriormente apresentados, podemos observar que nossos dados estão de acordo com a literatura. A alta concordância observada entre os dois métodos, em nosso estudo, pode ser justificada pelo fato de terem sido isoladas apenas cepas comumente identificadas na rotina laboratorial, a partir de amostras clínicas, não sendo isolada nenhuma cepa rara.

Segundo os estudos apresentados, a precisão do equipamento BD Phoenix na identificação de enterobactérias, em comparação ao método manual, varia de $89,9 \%$ a $100 \%$, com a utilização de cepas padrão $0^{3,4,9,10}$. No presente estudo apenas pudemos definir a concordância entre os métodos, e não a precisão, visto que não foi utilizada a identificação de cepas padrão, somente de amostras clínicas de pacientes hospitalizados.

Uma identificação precisa de microrganismos, até o nível de espécie, envolvidos num processo de doença é frequentemente essencial não só para a terapia antimicrobiana eficaz, mas também para a compreensão do início e da progressão da doença ${ }^{11}$. Nesse sentido, observamos melhor eficiência do método automatizado em relação ao método manual, pois em sete dos registros analisados as espécies não foram identificadas pelo método manual. E, apesar da identificação manual ter uma boa sensibilidade, a automação teve um melhor desempenho na identificação.

Os métodos automatizados oferecem vantagens como a reprodutibilidade, disponibilidade de gerar resultados dentro de um dia útil e liberação de quantidades reduzidas de contaminantes, Além disso, esses métodos apresentam um sistema de interfaciamento, que facilita a liberação de laudos e proporciona a oportunidade ao médico de obter resultados parciais ou integrais do exame de seu paciente ${ }^{12}$. As desvantagens são o custo elevado e a dependência de um sistema fechado, que exige manutenção e renovação de seus softwares e o treinamento do profissional que utiliza o equipamento. Ainda em relação à automação, a mesma é deficiente na identificação da motilidade das bactérias ${ }^{4,13,14}$.

No método manual com conjuntos de provas bioquímicas, é possível realizar a triagem e identificação presuntiva dos principais gêneros bacterianos de interesse clínico, sem caracterizar com precisão as respectivas espécies. Através desse método, em torno de $90 \%$ das enterobactérias isoladas de amostras clínicas são identificadas por meio de esquemas utilizando as tabelas de identificação, apresentando um percentual alto de discriminação. Os resultados são entregues entre 48 a 72 horas, tempo relativamente longo quando comparado com as automações ${ }^{15}$.

Sistemas automatizados têm como principais objetivos aumentar a eficácia e melhorar a relação custo beneficio da rotina laboratorial ${ }^{4}$. No laboratório em estudo, o custo efetivo da automação é muito superior ao método manual; porém, em se tratando de um laboratório inserido em um ambiente hospitalar, é importante a utilização de metodologias que apresentem boa precisão na identificação de microrganismos. Dessa forma, a identificação de bactérias através do método automatizado é realizada apenas em amostras provenientes de pacientes internados, 
concomitante ao método manual, o que justifica as amostras selecionadas no atual estudo.

As enterobactérias prevalentes no presente estudo apontam para uma representação realista dos microrganismos isolados em amostras clínicas provenientes de um ambiente hospitalar, sendo comum a predominância de Escherichia coli, Klebsiella spp. e Enterobacter spp. ${ }^{16,17}$. No estudo de Moura et al. ${ }^{18}$, evidenciou-se que o microrganismo causador do maior número de infecções em um ambiente hospitalar foi a Klebsiella pneumoniae $(35,46 \%)$, que teve uma prevalência semelhante à observada no laboratório em estudo ${ }^{18}$.
Como conclusão, o presente estudo demonstra uma boa concordância entre os métodos manual (bioquimismo) e automatizado (BD Phoenix) na identificação das principais enterobactérias isoladas em amostras clínicas. A deficiência na identificação de espécies sugere a utilização dos dois métodos, principalmente em pacientes hospitalizados, que apresentam frequentemente infecções por agentes multirresistentes. Estudos subsequentes com cepas raras e padronizadas poderão ser realizados para estabelecer uma comparação entre os métodos e determinar sua precisão.

\section{REFERÊNCIAS}

1. Khan F, Rizvi M, Shukla I, Malik A. A novel approach for identification of members of Enterobacteriaceae isolated from clinical samples. Biol Med. 2011;3(2):313-9.

2. Collee JG, Fraser AG, Marmion BP, Simmons A. Enterobacteriaceae: escherichia, klebsiella, proteus and other genera. In: Crichton PB, editor. Mackey and McCartney practical medical microbiology. New Delhi: Elsevier; 2006. p. 361-7.

3. Carroll KC, Glanz BD, Borek AP, Burger C, Bhally HS, Henciak $S$, et al. Evaluation of the BD Phoenix automated microbiology system for identification and antimicrobial susceptibility testing of Enterobacteriaceae. J Clin Microbiol. 2006;44(10):3506-9. http:// dx.doi.org/10.1128/JCM.00994-06. PMid:17021074

4. Snyder JW, Munier GK, Johnson CL. Direct comparison of the BD phoenix system with the MicroScan WalkAway system for identification and antimicrobial susceptibility testing of Enterobacteriaceae and nonfermentative gram-negative organisms. J Clin Microbiol. 2008;46(7):2327-33. http://dx.doi. org/10.1128/JCM.00075-08. PMid:18495856

5. Menozzi MG, Eigner U, Covan S, Rossi S, Somenzi P, et al. Twocenter collaborative evaluation of performance of the BD phoenix automated microbiology system for identification and antimicrobial susceptibility testing of gramnegative bacteria. J Clin Microbiol. 2006;44(11):4085-94. http://dx.doi. org/10.1128/JCM.00614-06. PMid:17005752
6. O'hara CM. Manual and automated instrumentation for identification of Enterobacteriaceae and other aerobic gram-negative bacilli. Clin Microbiol Rev. 2005;18(1):147-62. http://dx.doi. org/10.1128/CMR.18.1.147-162.2005. PMid:15653824

7. Ng SY, Kwang LL, Tan TY. Identification of Gram-negative bacilli directly from positive blood culture vials. J Med Microbiol. 2007;56(Pt 4):475-9. http://dx.doi.org/10.1099/ jmm.0.46708-0. PMid:17374886

8. Vargas LJ, Vila A, Lanza A, Bonvehi P, Nazar J, Mikietuk A, et al. Utilidad del sistema VITEK en la identificación bacteriana y estudios de sensibilidad antimicrobiana. Acta Bioquím Clín Latinoam. 2005;39(1):19-25.

9. O'Hara CM. Evaluation of the Phoenix 100 ID/AST system and NID panel for identification of Enterobacteriaceae, Vibrionaceae, and commonly isolated nonenteric gram-negative bacilli. J Clin Microbiol. 2006;44(3):928-33. http:// dx.doi.org/10.1128/JCM.44.3.928933.2006. PMid:16517878

10. Stefaniuk E, Baraniak A, Gniadkowski M, Hryniewicz W. Evaluation of the BD Phoenix automated identification and susceptibility testing system in clinical microbiology laboratory practice. Eur J Clin Microbiol Infect Dis. 2003;22(8):479-85. http://dx.doi. org/10.1007/s10096-003-0962-y. PMid:12884060

11. Spratt DA. Significance of bacterial identification by molecular biology methods. Endod Top. 2004;9(1):514. http://dx.doi.org/10.1111/j.1601 1546.2004.00106.x.

12. Donay JL, Mathieu D, Fernandes $P$, Prégermain $C$, Bruel $P$, Wargnier $A$, et al. Evaluation of the automated phoenix system for potential routine use in the clinical microbiology laboratory. J Clin Microbiol. 2004;42(4):1542-6. http://dx.doi. org/10.1128/JCM.42.4.15421546.2004. PMid:15071001

13. Junkins AD, Arbefeville SS, Howard WJ, Richter SS. Comparison of BD Phoenix AP Workflow with Vitek 2. J Clin Microbiol. 2010;48(5):1929-31. http://dx.doi.org/10.1128/JCM.0011110. PMid:20220158

14. Winstanley T, Courvalin P. Expert systems in clinical microbiology. Clin Microbiol Rev. 2011;24(3):515-56. http://dx.doi.org/10.1128/CMR.0006110. PMid:21734247

15. Agência Nacional de Vigilância Sanitária (ANVISA). Detecção e identificação de bactérias de importância médica - Módulo V. Brasília: ANVISA; 2004.

16. Ocaña Carrizo AV, Rocchi M, Gasparotto A, Conrero I, Navarro $\mathrm{M}$, Factorovich S, et al. Bacteriemia por enterobacterias en adultos en un hospital universitario: análisis de cinco años. Rev Argent Microbiol. 2007;39(1):38-43. PMid:17585658.

17. Nogueira PSF, Moura ERF, Costa MMF Monteiro WMS, Brondi L. Perfil da infecção hospitalar em um hospital universitário. Rev Enferm UERJ. 2007;17(1):96-101.

18. Moura ME, Campelo SM, Brito FC, Batista OM, Araújo TM Oliveira AD. Infecção hospitalar: estudo de prevalência em um hospital público de ensino. Rev Bras Enferm. 2007;60(4):416-21. http://dx.doi.org/10.1590/S003471672007000400011 . PMid:18041525 